University of Nebraska - Lincoln

DigitalCommons@University of Nebraska - Lincoln

December 1985

\title{
Magnetic transitions and scaling behavior in Gd-rich glasses
}

\author{
M.J. O'Shea \\ Cardwell Hall, Department of Physics, Kansas State University, Manhattan, Kansas \\ David J. Sellmyer \\ University of Nebraska-Lincoln, dsellmyer@unl.edu
}

Follow this and additional works at: https://digitalcommons.unl.edu/physicssellmyer

Part of the Physics Commons

O'Shea, M.J. and Sellmyer, David J., "Magnetic transitions and scaling behavior in Gd-rich glasses " (1985). David Sellmyer Publications. 145.

https://digitalcommons.unl.edu/physicssellmyer/145

This Article is brought to you for free and open access by the Research Papers in Physics and Astronomy at DigitalCommons@University of Nebraska - Lincoln. It has been accepted for inclusion in David Sellmyer Publications by an authorized administrator of DigitalCommons@University of Nebraska - Lincoln. 


\title{
Magnetic transitions and scaling behavior in Gd-rich glasses
}

\author{
M. J. O'Shea \\ Cardwell Hall, Department of Physics, Kansas State University, Manhattan, Kansas 66506 \\ D. J. Sellmyer \\ Behlen Laboratory of Physics, University of Nebraska, Lincoln, Nebraska 68588
}

(Received 8 April 1985)

\begin{abstract}
The magnetic properties of amorphous alloys with composition $\mathrm{Gd}_{x} \mathrm{La}_{72-x} \mathrm{Ga}_{18} \mathrm{~B}_{10}$ have been investigated using ac-susceptibilty and dc-magnetization techniques in the temperature range 4.2-300 $\mathrm{K}$. This system shows double-transition behavior for $x \geq 67$ and paramagnetic-spin-glass transition behavior for $x<67$. Time-dependent magnetization effects are present in the spin-glass phase and these effects vanish in finite fields, in qualitative agreement with mean-field theory. The magnetic isotherms around both the paramagnetic-ferromagnetic and ferromagnetic-spin-glass transitions scale according to the scaling hypothesis, and these results are discussed in some detail. A magnetic phase diagram for this system is obtained from the transition temperatures determined from scaling.
\end{abstract}

\section{INTRODUCTION}

Alloy systems with competing exchange interactions have been the subject of much research in recent years. ${ }^{1,2}$ Many of these systems show double-transition or paramagnetic-to-spin-glass behavior depending on composition. The double-transition behavior is often called reentrant behavior and represents a paramagnetic (PM) -ferromagnetic (FM) -spin-glass (SG) series of transitions with decreasing temperature. Such behavior exists in a number of amorphous transition-metal ${ }^{3-6}$ and $\mathrm{Gd}$ systems, ${ }^{7-9}$ as well as the crystalline systems $\mathrm{Au}-\mathrm{Fe},^{10}$ Ni-Mn, ${ }^{11}$ and insulating Eu-Sr-S. ${ }^{1}$ The exchange interactions in these systems vary from indirect in Gd-rich systems to partly direct in transition-metal systems to superexchange in Eu-Sr-S, and the common denominator in all of these systems is a random frustration in the microscopic interactions.

A number of experiments including the presence of an essentially static peak in the dc susceptibility independent of cooling rate $\mathrm{e}^{12}$ suggest a phase transition description of the PM-SG transition. Simulations of the $d$ (dimensionality) $=2$ Ising model with short-range interactions ${ }^{13}$ reproduced much of the observed nonequilibrium SG behavior; however, this model does not undergo a phase transition at finite temperatures. Some recent simulations and calculations for this model with $d=3,{ }^{14}$ however, indicate a continuous phase transition, so that the PM-SG transition seen experimentally may be a continuous phase transition. Simulations of short-range Heisenberg spin systems which are expected to be closer to reality are unable to reproduce the observed nonequilibrium SG behavior unless anisotropic interactions are included. ${ }^{15,16}$ Such simulations show that anisotropy is an important factor in determining SG behavior in real systems.

The double-transition behavior seen in experiment has been somewhat more puzzling. Simulations of systems with short-range interactions have so far failed to repro- duce such behavior. The presence of nonequilibrium SG behavior at low temperatures, though, suggests that anisotropy is required to produce such behavior. It is generally accepted now that the PM-FM transition seen in amorphous alloys represents a true continuous magnetic phase transition analogous to those seen in nonrandom systems. The scaling behavior at this transition follows the scaling hypothesis in all of the systems studied so far (see, for example, Refs. 17 and 18). In the absence of any theoretical predictions to compare to, the scaling hypothesis has been applied to the FM-SG transition to determine whether this transition follows the scaling hypothesis, as one would expect for a continuous phase transition. A scaling of the magnetization has been shown to be possible at this transition by a number of workers. ${ }^{3-6}$

The Sherrington-Kirkpatrick solution ${ }^{19}$ of a (replica symmetric) Ising mean-field exchange-fluctuation model shows double-transition behavior. In the SG phase and part of the FM phase, however, replica symmetry is bro$\mathrm{ken}^{20}$ and a functional order parameter ${ }^{21}$ is required to describe the resulting low-temperature state. A finite field $H_{c}$ is required to stabilize the Sherrington-Kirkpatrick solution and, for $T \gtrsim T_{p g}, H_{c} \sim\left(T-T_{p g}\right)^{3 / 2}$. This demarcation line has become known as the de Almeida-Thouless line, and the dynamical theory of Sompolinsky ${ }^{22}$ shows that this line marks the onset of nonequilibrium behavior including irreversibility. Surprisingly good agreement has been found between this mean-field prediction and experiment in a number of frustrated systems, including Fe-Mn glasses $^{23}$ and Eu-Sr-S. ${ }^{24}$ Extension of these mean-field calculations to Heisenberg spins which are expected to be somewhat closer to reality produced some additional transitions and magnetic states. In particular, a mixed FMSG state in which longitudinal FM and transverse SG order coexist is predicted at low temperatures. ${ }^{25}$ There are actually two such mixed phases with varying degrees of broken replica symmetry. In this model the spontaneous moment does not go to zero below the FM phase, so that 
no double-transition behavior is present.

In this work we shall discuss the magnetic transition behavior of Gd-rich glasses of the form $\mathrm{Gd}_{x} \mathrm{La}_{72-x} G_{28}$, where $G_{28}$ represents $G_{18} B_{10}$ and is used as a glass former. Experimental techniques are discussed in Sec. II. This system shows double-transition and PM-SG-transition behavior, depending on composition $x$. This phase diagram has been extended and modified from that in Ref. 8 and is discussed in Sec. III. We have previously discussed the behavior of the thermoremanent (TRM) and isothermal remanent (IRM) magnetizations and their associated finite-field magnetizations. ${ }^{26}$ Both of these remanent magnetizations and $M(H)$, measured in increasing field after zero-field cooling, show a dependence on time; these effects are also discussed in Sec. III. The field-cooled magnetization does not show a time dependence on a timescale of $\sim 15 \mathrm{~min}$ and extrapolates smoothly to zero, indicating the absence of a macroscopic moment. No evidence was found for a mixed FM-SG phase with longrange longitudinal FM order. In real systems some anisotropy such as dipolar or Dzaloshinski-Moriya anisotropy is present. It is known that off-diagonal components of these interactions in a possible mixed FM-SG state can couple the longitudinal and transverse spin components and destroy the longitudinal FM order. ${ }^{27}$ The absence of such a mixed FM-SG phase in Ref. 26 is therefore not too surprising. The scaling behavior and critical exponents are presented in Sec. IV and results for the FM-SG transition are presented in Sec. V. Finally in Sec. VI a discussion of our results and our conclusions are presented.

\section{EXPERIMENT}

The samples of this work were made by splat-cooling arc-melted droplets of the appropriate alloy composition. $\mathrm{X}$-ray diffractograms using Mo $K \alpha$ radiation show the samples to be amorphous over the complete compositional range $x=72$ to $x=0$. Examples of x-ray diffractograms for the $x=68$ and 54 alloys are shown in Fig. 1. The samples were in the form of sandwiches of $\sim 6$ strips, each $1 \mathrm{~cm}$ long and $\sim 50 \mu \mathrm{m}$ thick, and were oriented parallel to the applied field in order to minimize demagnetization effects.

ac-susceptibility measurements at $280 \mathrm{~Hz}$ with an $\mathrm{rms}$ amplitude of 0.1 Oe were made in both zero and finite dc magnetic fields up to $400 \mathrm{Oe}$ in the temperature range 4.2 to $300 \mathrm{~K}$. Magnetization measurements were made using a vibrating-sample magnetometer (VSM) described in a previous publication ${ }^{28}$ in the temperature range of 4.2 to $300 \mathrm{~K}$. High magnetic fields up to $80 \mathrm{kOe}$ were supplied by a superconducting magnet. At low fields the superconducting magnet showed some hysteresis in its currentversus-field characteristic and a residual field of $\sim 20 \mathrm{Oe}$ in the open-circuit state. It was found that this hysteresis appeared after cycling the magnet up to $80 \mathrm{kOe}$, but for low-field cycling $(<1 \mathrm{kOe})$ the residual field is less than 2 Oe. For this reason low-field measurements were made first after the initial cooldown of the superconducting magnet and high-field measurements were made only when these low-field measurements were completed.

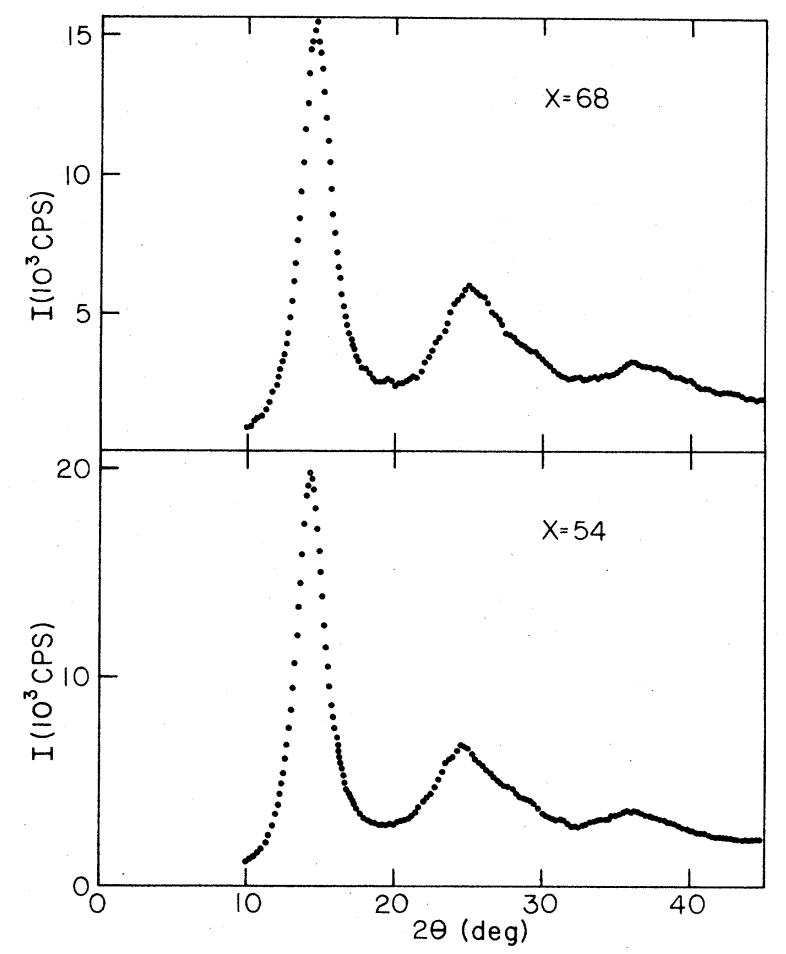

FIG. 1. X-ray diffractograms for the $x=68$ and $x=54$ samples using Mo $K \alpha$ radiation. The ordinate units are in $10^{3}$ counts per second.

\section{MAGNETIC PHASE DIAGRAM AND SPIN-GLASS BEHAVIOR}

The magnetic phase diagram of the $\mathrm{Gd}_{x} \mathrm{La}_{72-x} G_{28}$ system is shown in Fig. 2. This diagram has been extended to lower $x$ from that in Ref. 8 and the position of the FM-SG is somewhat modified. This diagram shows regions of PM, FM, and SG behavior, and for $x \geq 67$ double-transition behavior is present. The SG region in these concentrated $\mathrm{Gd}$ alloys may more accurately be described as a cluster SG. If $x<67$ only PM-SG behavior

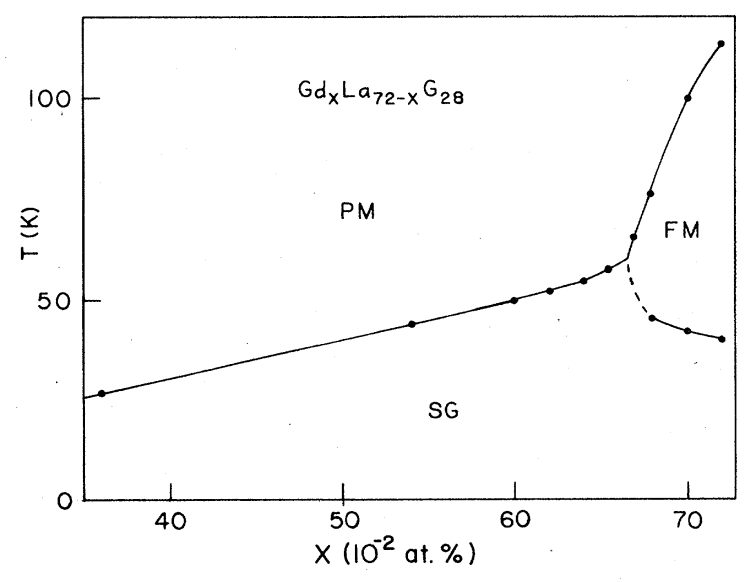

FIG. 2. Phase diagram of the behavior of the Gd-La system. 
is present. The effect of increasing the La content (decreasing $x$ ) is to generally increase frustration effects. The position of the PM-FM and PM-SG lines were determined from ac-susceptibility measurements. The ac susceptibility was checked for any dependence on frequency between 2.8 and $280 \mathrm{~Hz}$ and none was found, as may be seen from Fig. 3. The solid line marking the FM-SG boundary was determined from a scaling analysis (Sec. V) around this transition, and the transition temperature is very close to the peak corresponding to the FM-SG transition in the out-of-phase part of the ac susceptibility, an example of which is shown in Fig. 3 for the $x=72$ sample. The experimental multicritical point is at a much higher concentration in this $\mathrm{Gd}$ system than in transition-metal glasses involving $\mathrm{Fe}-\mathrm{Ni}$ and $\mathrm{Co}-\mathrm{Ni}$, where the experimental multicritical point is at $\sim 16$ at. $\% \mathrm{Fe}$ and $\sim 35$ at. $\% \mathrm{Co}$, respectively. ${ }^{3} \mathrm{Fe}-\mathrm{Mn}$ glasses have an experimental multicritical point at $\sim 66$ at. \% Fe which is close to the system of this work. In this system Mn contributes significant antiferromagnetic exchange interactions, and the phase diagram similarities suggest that significant antiferromagnetic interactions are also present in the Gd system of this work. We also note that the percolation threshold for binary Gd-nonmagnetic-metal amorphous alloys generally lies between 15 and 30 at. \% $\mathrm{Gd},{ }^{29}$ which is some distance below our experimental multicritical point.

In applied dc fields the double-transition behavior may be resolved in the ac susceptibility. Such experiments have been discussed by Geohegan and Bhagat. ${ }^{30}$ Figures 4(a) and 4(b) show this behavior for the $x=72$ and 68 samples. It can be seen that the applied field shifts the PM-FM transition to higher temperatures. In the $x=64$ sample [Fig. 4(c)], where only a PM-SG transition is present in zero dc fields, the effect of an applied field is to reduce the size of this peak and to shift its position to lower temperatures.

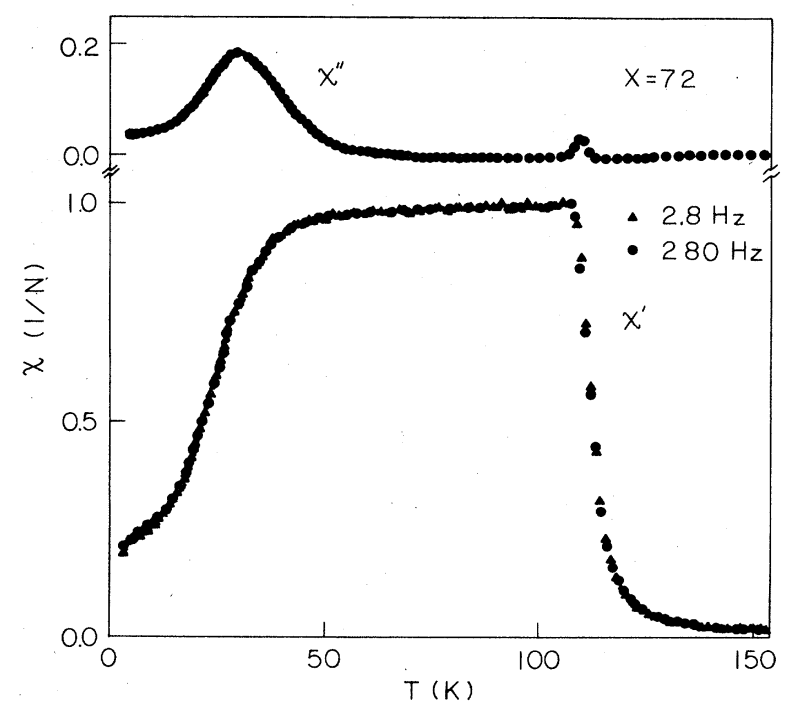

FIG. 3. The ac susceptibility $\chi^{\prime}$ (in-phase) of the $x=72$ sample as a function of frequency. The out-of-phase part of the ac susceptibility $\chi^{\prime \prime}$ is also shown.
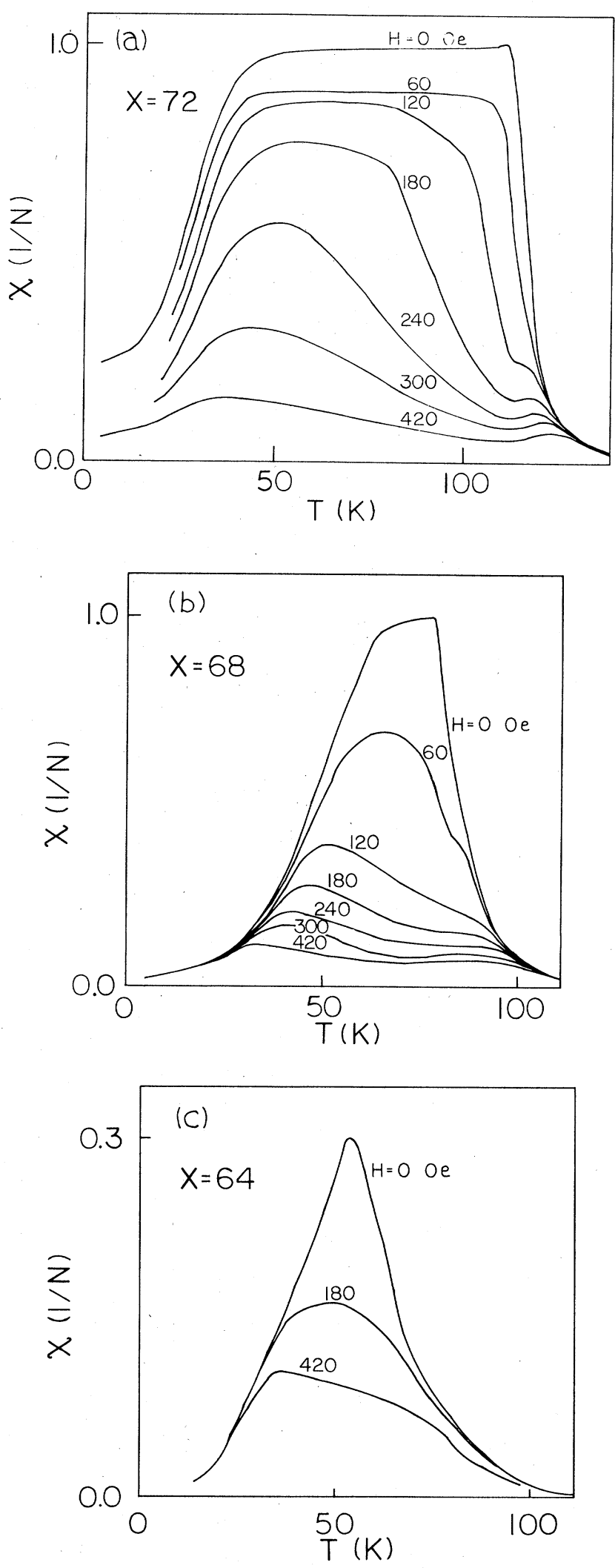

FIG. 4. The ac susceptibility of the (a) $x=72$, (b) $x=68$, and (c) $x=64$ samples in zero and nonzero applied dc magnetic fields. 


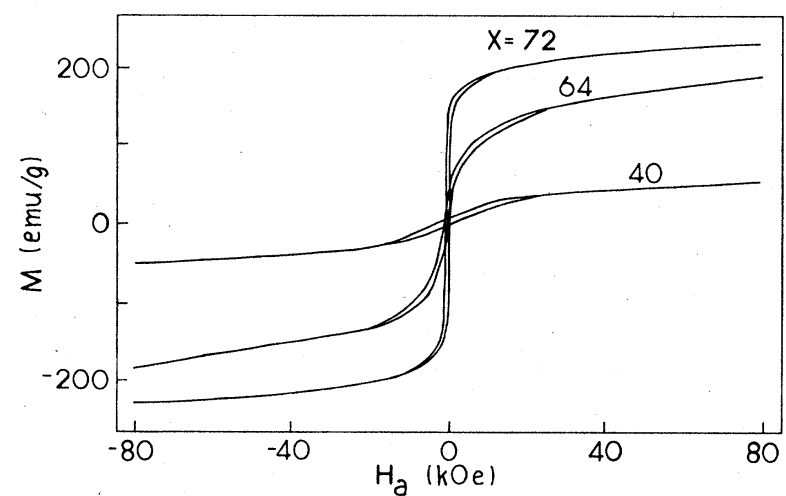

FIG. 5. Hysteresis loops up to $80 \mathrm{kOe}$ for the $x=72,64$, and 40 samples.

In the SG state hysteresis is present for all of the compositions studied, as may be seen in Fig. 5 for our $x=72$, 64 , and 40 samples. In the FM state of the $x=72$ and 68 samples the hysteresis is too small to measure (the coer-
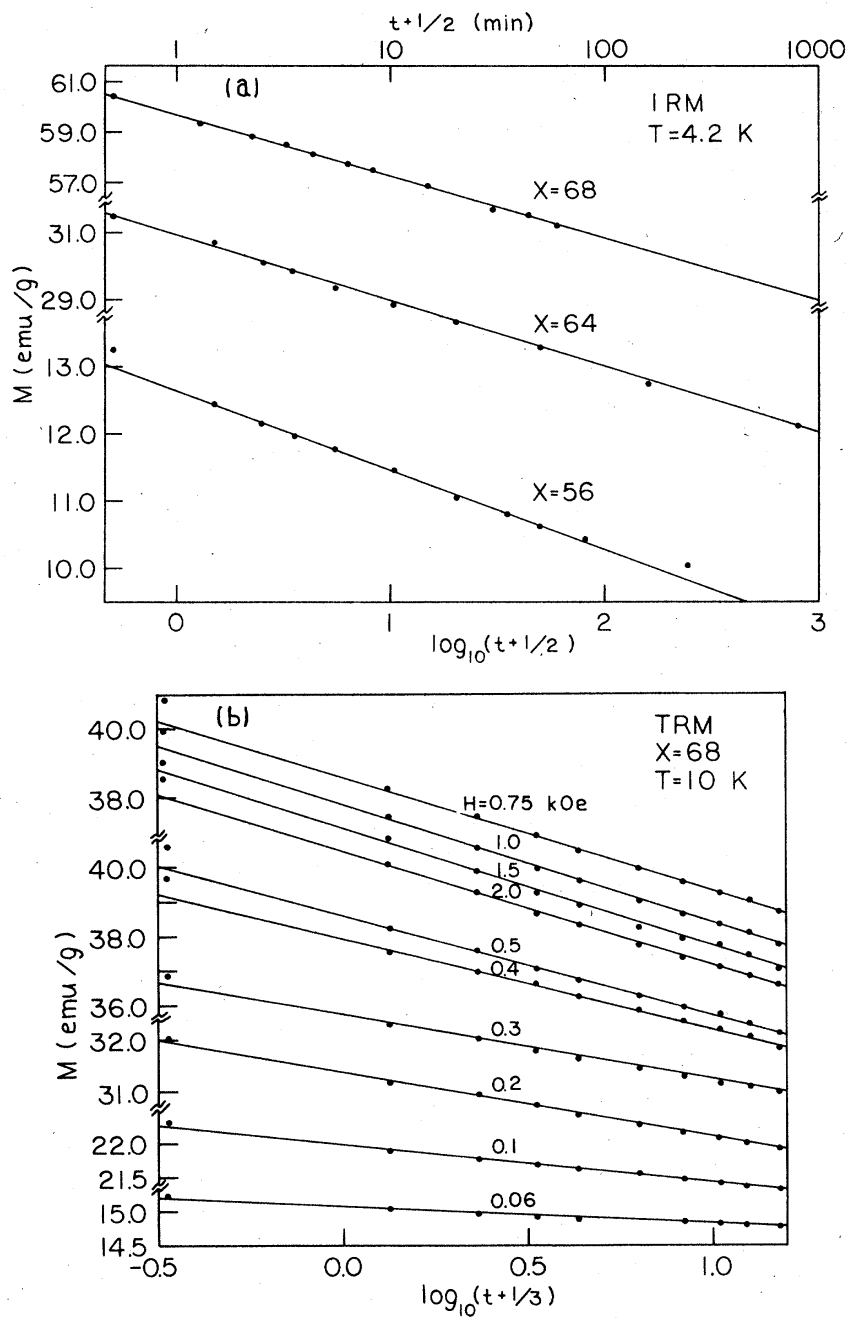

FIG. 6. The time dependence of the (a) IRM after cycling the field to $80 \mathrm{kOe}$ for the $x=68,64$, and 56 samples at $4.2 \mathrm{~K}$, and (b) TRM at $10 \mathrm{~K}$ in the $x=68$ sample as a function of fieldcooling field. civity is less than $2 \mathrm{Oe}$ ). At $4.2 \mathrm{~K}$ the coercive field for the $x=72$ sample is 250 Oe and shows a gradual increase with decreasing Gd concentration. Also, as the concentration of $\mathrm{Gd}$ decreases, it becomes increasingly difficult to saturate the magnetization. Curvature is present in the less concentrated glasses at our highest fields, most likely due to the presence of significant antiferromagnetic interactions. These glasses also show isothermal remanent magnetization and thermoremanent magnetization typical of many SG systems, and these results have been discussed in a previous publication. ${ }^{26}$ The IRM and TRM, and also the initial magnetization taken with increasing field after zero-field cooling, all show a time dependence at low temperatures, which conforms well to an empirical logarithmic behavior of the form $M=M_{0}$ $+S(H, T) \log _{10}\left(t+t_{0}\right)$ on our timescale $\left(\frac{1}{2}-800 \mathrm{~min}\right)$. The quantity $S$ gives a measure of the strength of time dependence, and $t_{0}$ is a constant which is found experimentally to be approximately equal to the time elapsed between reducing the field to zero and taking the first measurement of magnetization as a function of time. Figure 6(a) shows the time dependences of the IRM after cycling the applied field to $80 \mathrm{kOe}$ and back to zero after zerofield cooling to $4.2 \mathrm{~K}$ for the $x=68,64$, and 56 samples. Figure $6(\mathrm{~b})$ shows the time dependence of the TRM in the $x=68$ sample at $10 \mathrm{~K}$ as a function of the field-cooling field. The values of $S(H, T)$ determined from the slope of such data for the IRM, TRM, and also $M(H)$ are shown for the $x=68$ sample in Figs. 7(a) and 7(b), respectively. $S$ for the $M(H)$ curve shows a large maximum and goes rapidly to a small value at higher fields, indicating the vanishing of time-dependent effects. Such behavior is
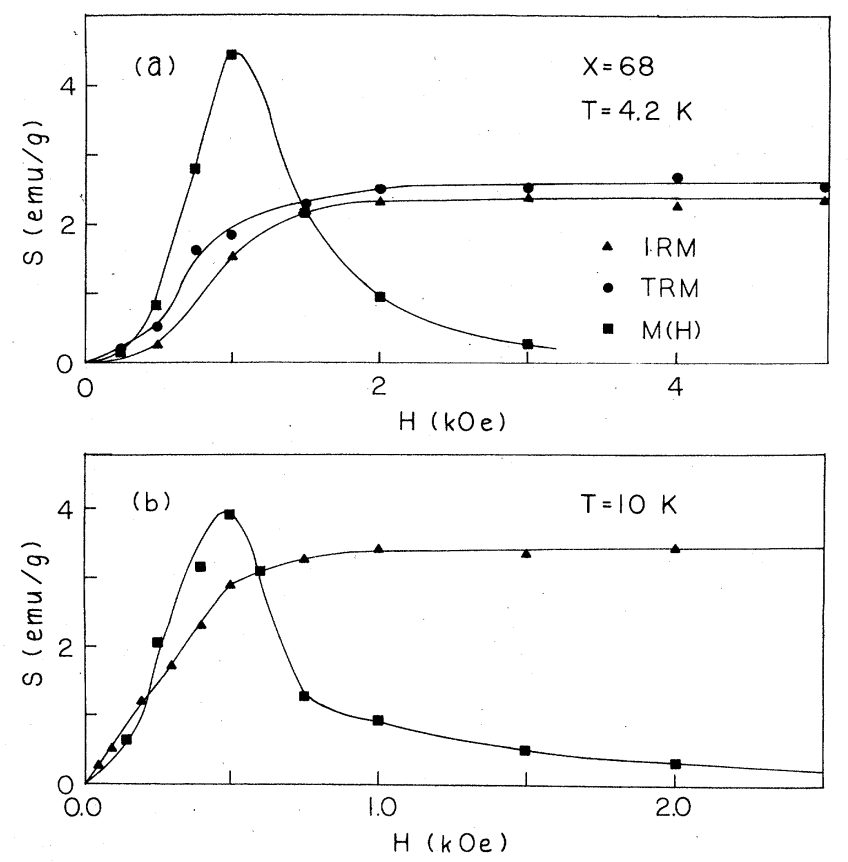

FIG. 7. The value of the coefficient $S$ for the IRM, TRM, and initial magnetization as a function of applied field in the $x=68$ sample at (a) $4.2 \mathrm{~K}$ and (b) $10 \mathrm{~K}$. 


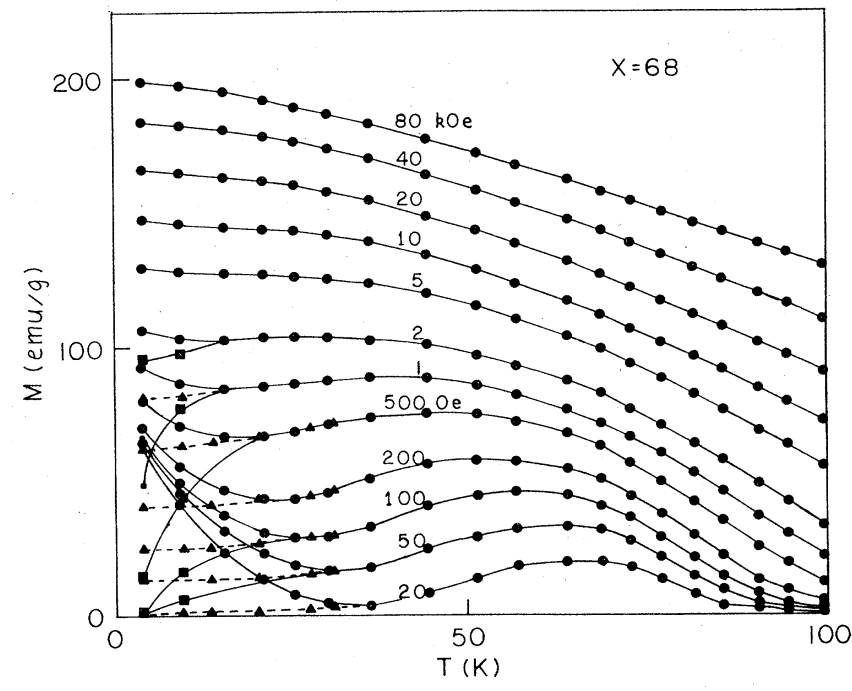

FIG. 8. Initial magnetization ( $\square$ ), field-cooled magnetization $(\Delta)$, and magnetization taken with decreasing field $(\bullet)$ in the $x=68$ sample as a function of temperature.

also seen in the onset of hysteresis in the temperature dependence of the magnetization. Figure 8 shows the initial magnetization, field-cooled magnetization, and magnetization taken with decreasing field at a given temperature. The onset of hysteresis occurs where these curves separate, and in both the $x=72$ and 68 glasses the temperature below which hysteresis is present decreases monotonically as the applied field increases. Also, as $x$ decreases and frustration effects become stronger, the region of hysteresis in the $H-T$ plane becomes larger. Such behavior has been seen in other SG systems and follows qualitatively the predictions of the replica-symmetrybreaking mean-field theory of de Almeida and Thouless. ${ }^{20}$
The onset of hysteresis as a function of $H, T$ agrees within experimental error with that determined from the onset of hysteresis in the IRM and TRM, and these results will be discussed in more detail in a later publication.

\section{CRITICAL BEHAVIOR AT THE PM-FM TRANSITION}

The magnetic equation of state in the critical region according to the scaling hypothesis may be written in the form $^{31}$

$$
m=m(\epsilon /|\epsilon|, h),
$$

where

$m=M /|\epsilon|^{\beta}, h=H /|\epsilon|^{\beta \delta}, \quad \epsilon=\left(T-T_{c}\right) / T_{c}$.

Here, $m, h$, and $\epsilon$ represent reduced magnetization, reduced magnetic field, and reduced temperature, respectively, and $T_{c}$ is the transition temperature of the transition of interest. The critical exponents $\beta, \delta$, along with other useful critical exponents, are defined by the following relations:

$$
\begin{aligned}
& C_{H} \sim|\epsilon|^{-\alpha}, \\
& M \sim|\epsilon|^{\beta}, \epsilon<0 \\
& \chi \sim|\epsilon|^{-\gamma}, \epsilon>0 \\
& M \sim H^{1 / \delta}, \quad \epsilon=0 .
\end{aligned}
$$

According to the scaling hypothesis, only two of these exponents are independent and, therefore, required to describe the static critical behavior. The reduced magnetic equation of state has two branches $\epsilon /|\epsilon|=+1,-1$ corresponding to $T>T_{c}$ and $T<T_{c}$, respectively. If the magnetization and magnetic field are plotted in reduced form, the magnetic isotherms should collapse onto two branches corresponding to $T>T_{c}$ and $T<T_{c}$.

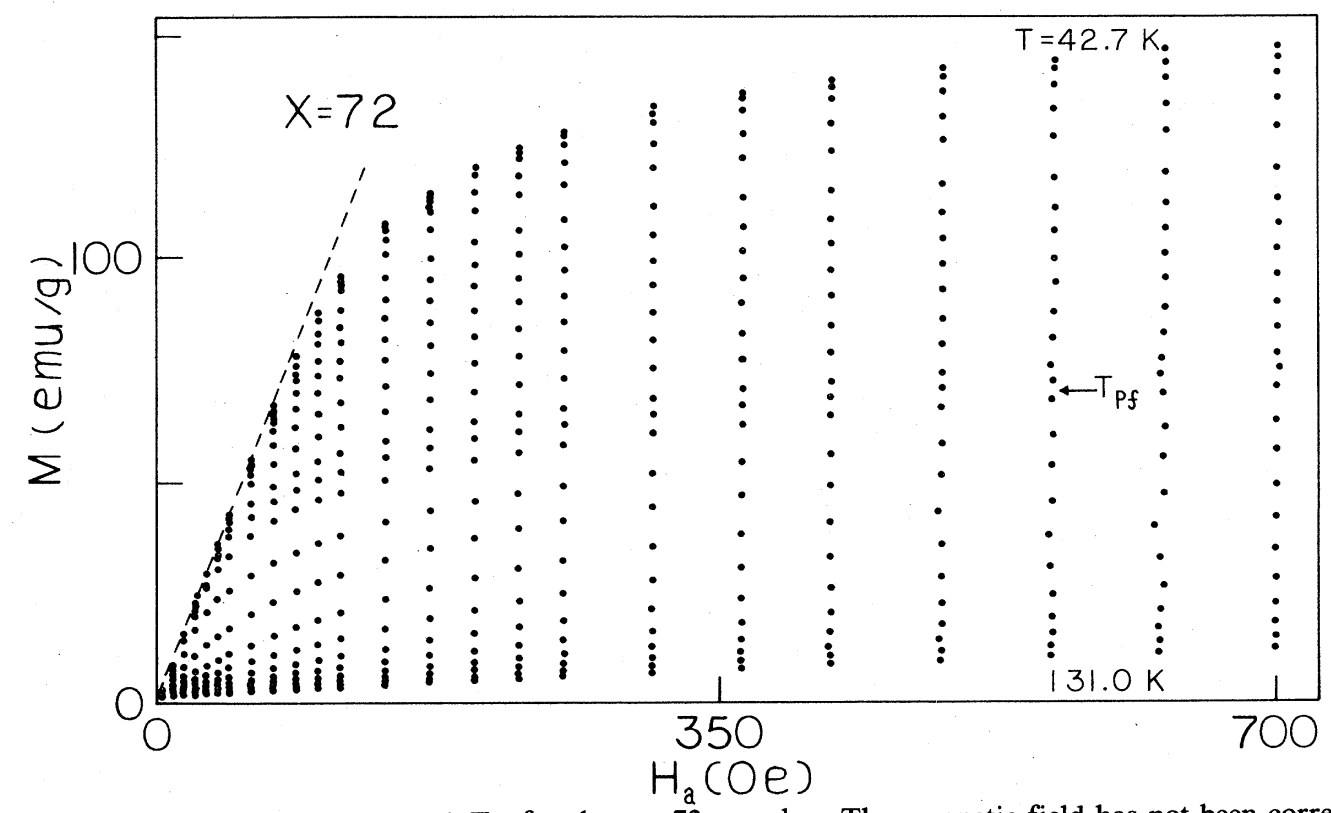

FIG. 9. Low-field magnetic isotherms around $T_{p f}$ for the $x=72$ samples. The magnetic field has not been corrected for demagnetization effects. 
In this section we discuss our results for the transition behavior at the PM-FM transition at $T_{c}=T_{p f}$ in our $x=72$ and 68 samples. As discussed in Sec. II, separate measurements were made in the field regions 2 to $700 \mathrm{Oe}$ and 1 to $80 \mathrm{kOe}$. Figure 9 shows the low-field magnetic isotherms and Fig. 10 shows the intermediate-field and low-field data for the $x=72$ sample plotted in reduced units. As may be seen, the quality of scaling is good, with the isotherms showing a well-defined collapse onto two branches. For the low-field data $\log _{10}(m)$ versus $\log _{10}(h)$ is plotted to show the scaling at the lowest fields. The exponents $\beta$ and $\delta$ were determined by varying each in small increments in the ranges $0.3<\beta<0.5,2<\delta<6$, and $T_{p f}$ was determined from susceptibility measurements and varied within its experimental error to obtain best collapse of the isotherms. The errors on $\beta, \delta$, and $T_{p f}$ represent the ranges over which good collapse of the magnetic iso-
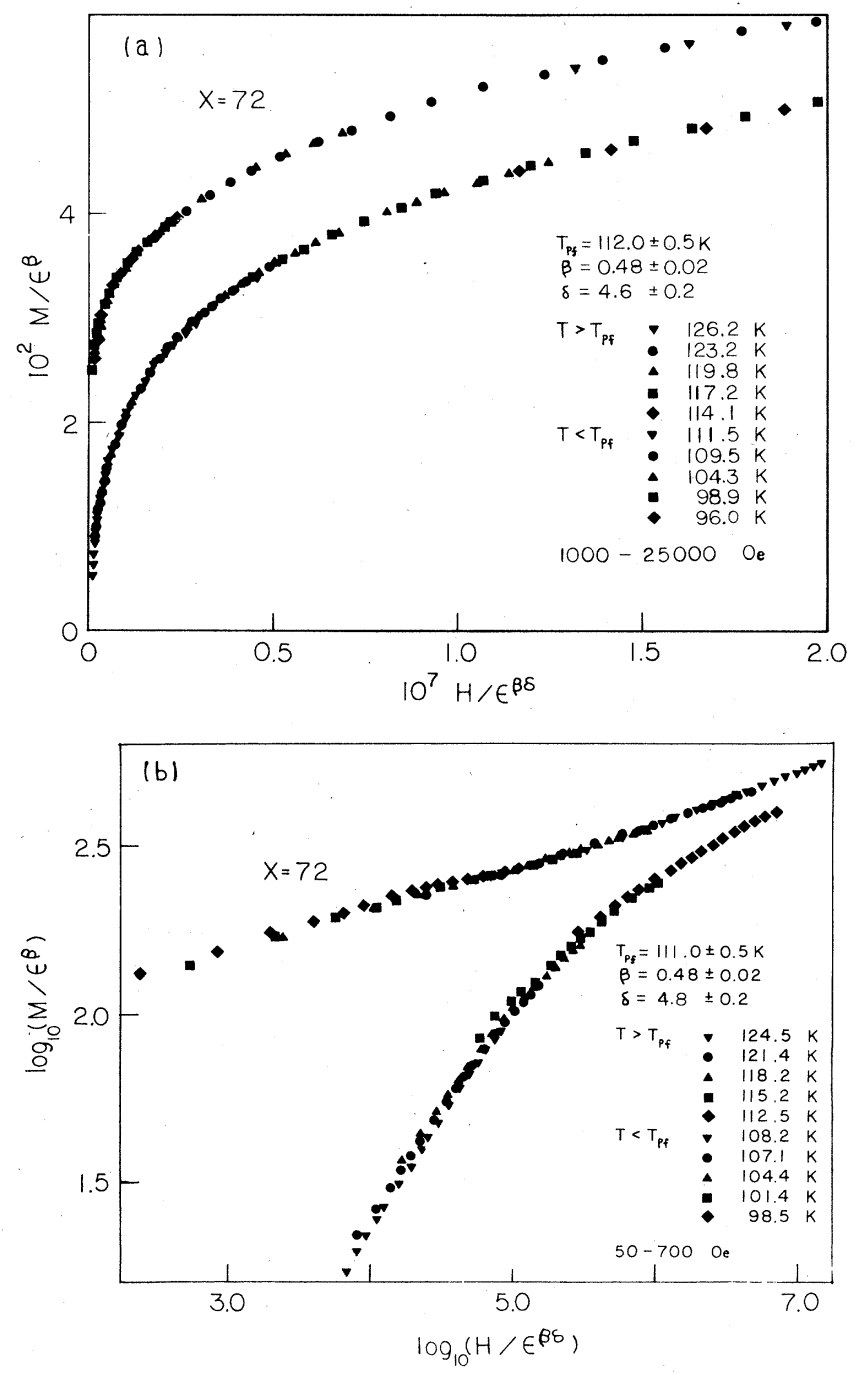

FIG. 10. (a) High-field and (b) low-field magnetization versus magnetic field for the $x=72$ sample around the PM-FM transition in reduced units (see text). The low-field data are plotted in logarithmic form to resolve the scaling behavior at the lowest fields. therms were obtained. The values of $\beta, \delta$, and $T_{p f}$ agree within experimental error for both sets of data. Demagnetization effects were negligible for the intermediate-field data, but become important at our lowest fields. The demagnetization factor $N$ was determined from the limiting low-field slope of magnetization versus temperature, and the internal field $H$ is calculated using $H=H_{a}-N M$, where $H_{a}$ is the applied magnetic field. Figure 9 shows our magnetic isotherms plotted against applied field at low fields for the $x=72$ sample. The dashed line represents our limiting low-field slope $(1 / N)$. The measured $N$ is consistent with the dimensions of the samples and varies by approximately 5\% from sample to sample. For samples with composition $x<68$ but still in the double-transition region $(x \geq 67)$, the susceptibility did not reach the demagnetization limit. Such behavior has also been found by other workers in transition-metal systems. Figure 11 shows the low-field magnetization around the PM-FM transition in the $x=68$ sample in reduced units. The collapse of the isotherms here is not as well defined as for the $x=72$ sample, even though demagnetization effects were found to be less important here. The critical exponents were determined in a similar manner as those in our $x=72$ sample. These exponents are given in Table I along with those previously measured in Gd glasses. In our low-field data significant deviations from scaling were present below 50 Oe for both our $x=72$ and 68 samples, presumably due to a combination of an inadequate demagnetization correction at the lowest fields and domain formation. We have also measured the exponent $\gamma$ directly using ac susceptibility for the $x=72$ sample, and a least-squares fit to $\log _{10} \chi$ versus $\log _{10} \epsilon$ yields $\gamma=1.60 \pm 0.04$ (see Fig. 12). This agrees within experimental error with the value of $\gamma$ calculated from $\beta$ and $\delta$ of scaling (see Table I) using the scaling equality $\gamma=\beta(\delta-1) .^{31}$

The value of $\alpha$ may be calculated from $\beta, \delta$ using the

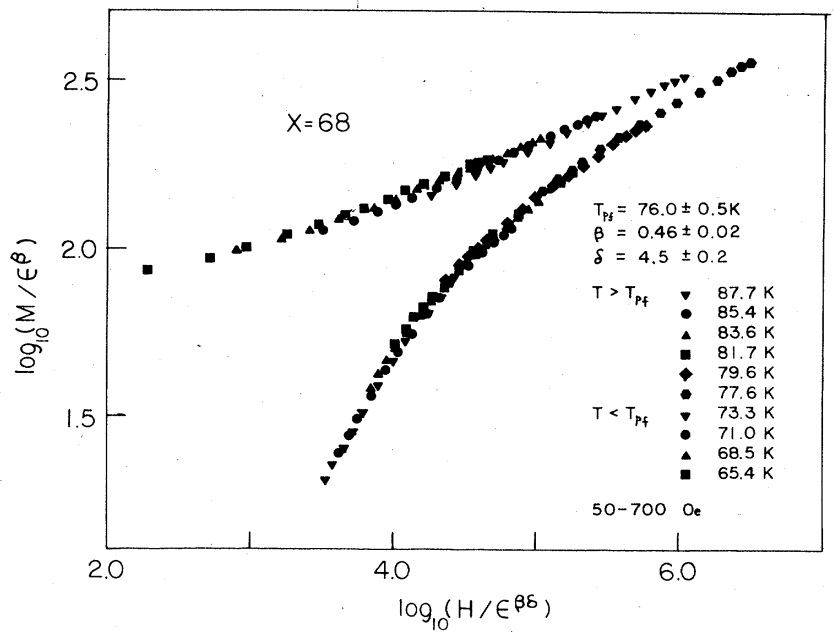

FIG. 11. Low-field magnetization versus magnetic field for the $x=68$ sample in reduced units (see text). The data are plotted in logarithmic form to resolve the scaling at the lowest fields. 


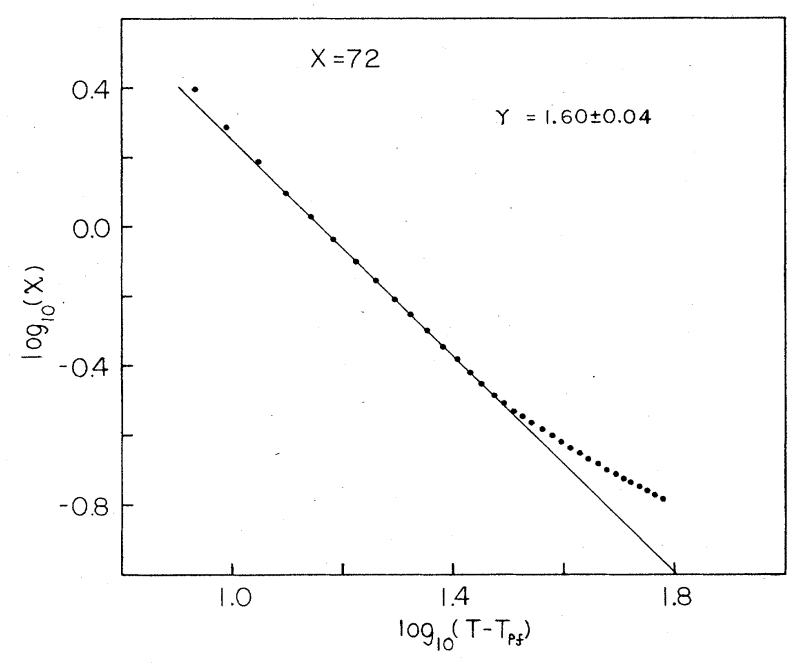

FIG. 12. The ac susceptibility in logarithmic form for the $x=72$ sample for $T>T_{p f}$. The solid line represents a leastsquares fit to the linear portion.

scaling hypothesis equality $\alpha=2-\beta(\delta+1)$, and it is given in Table I. It has been shown that sharp transition behavior for a disordered $n$-component system with $1<n<4$ is only possible when $\alpha<0 .{ }^{34}$ The values of $\alpha$ for this work are indeed less than zero, as has been found in a number of other metallic glasses.

\section{CRITICAL BEHAVIOR AT THE FM-SG TRANSITION}

It is not known yet whether the FM-SG transition at $T_{f g}$ represents a true phase transition. No model incorporating short-range frustrated interactions has thus far been able to reproduce the double-transition behavior observed in these and other magnetic systems. In the absence of any detailed model predictions to test against experiment close to $T_{f g}$, a number of workers ${ }^{3-6}$ have used the scaling hypothesis previously applied to the PM-FM phase transition to characterize this transition. We have followed earlier workers by doing a scaling analysis on the initial magnetization at this transition. Measurable hysteresis appears below $\sim 20 \mathrm{~K}$ in both the $x=72$ and 68 samples, and only magnetic isotherms taken above $20 \mathrm{~K}$ were used in our scaling analysis. In the case of the FM-
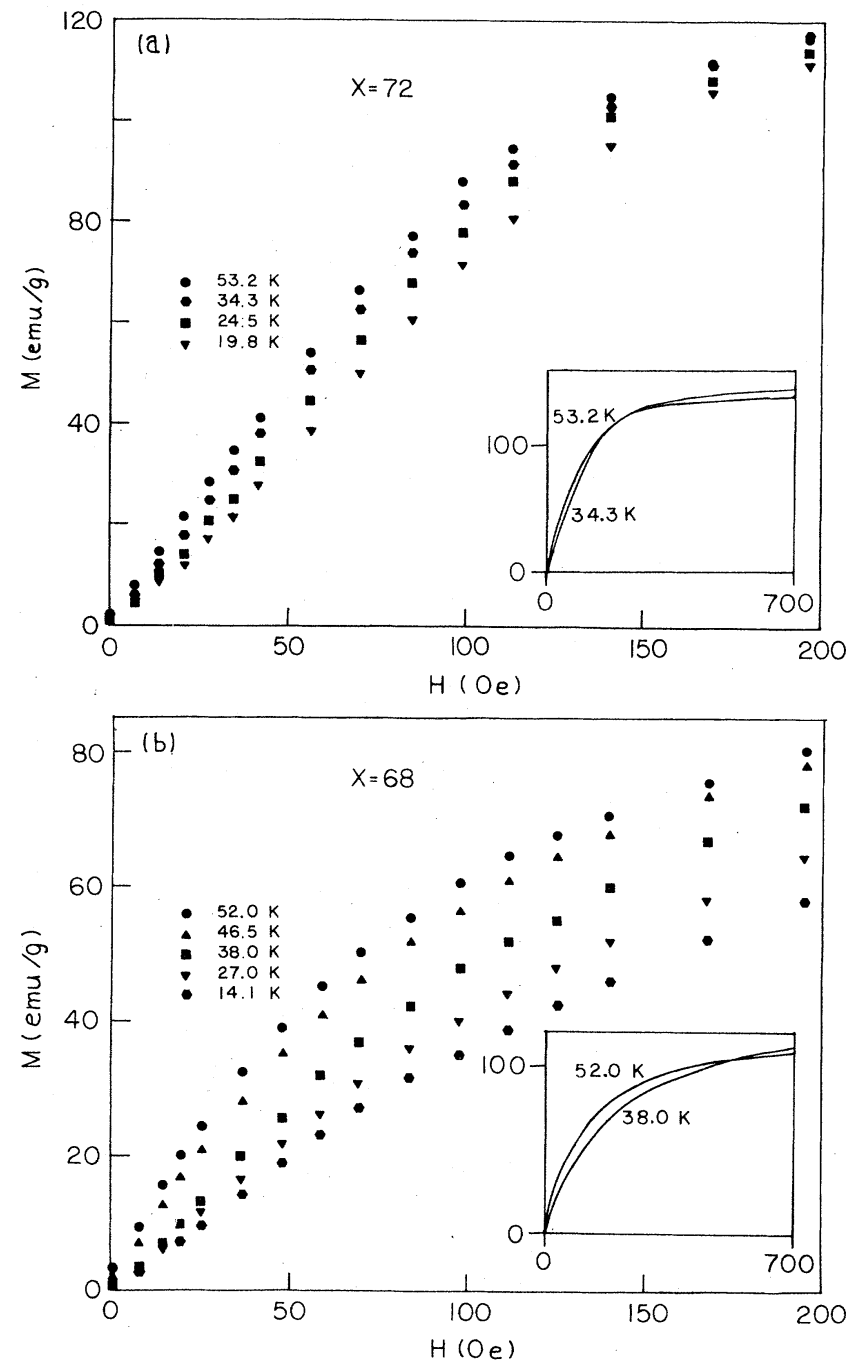

FIG. 13. Selected magnetic isotherms for the (a) $x=72$ and (b) $x=68$ samples at low fields around the FM-SG transition. The insets show the crossover of the isotherms to the usual high-field behavior, as discussed in the text.

SG transition the behavior of the magnetic isotherms as a function of temperature is reversed compared to those at the PM-FM transition. This may be seen for the case of the $x=72$ and 68 samples, for which a few isotherms are shown in Fig. 13. The initial magnetization at a given

TABLE I. Experimental critical exponent values in Gd-rich metallic glasses at the PM-FM transition. The symbol $G_{28}$ represents the combination $\mathrm{Ga}_{18} \mathrm{~B}_{10}$.

\begin{tabular}{llcccc}
\hline \hline & \multicolumn{1}{c}{$\alpha^{\mathrm{a}}$} & $\beta$ & $\gamma$ & \multicolumn{1}{c}{$\delta$} & $T_{p f}(\mathrm{~K})$ \\
\hline $\mathrm{Gd}_{80} \mathrm{Au}_{20}{ }^{\mathrm{b}}$ & $-0.17(9)$ & $0.44(2)$ & $1.29(5)$ & $3.96(3)$ & $149.4(2)$ \\
$\mathrm{Gd}_{2} \mathrm{Co}^{\mathrm{c}}{ }^{\mathrm{d}}$ & $\sim 0$ & $0.41(2)$ & $1.16(5)$ & $3.60(10)$ & $172(3)$ \\
$\mathrm{Gd}_{72} G_{28}{ }^{\mathrm{d}}$ & -0.74 & $0.48(2)$ & $1.60(4)$ & $4.7(2)$ & $112(1)$ \\
$\mathrm{Gd}_{68} \mathrm{La}_{4} G_{28}{ }^{\mathrm{d}}$ & -0.53 & $0.46(2)$ & & $4.5(2)$ & $76.0(5)$ \\
\hline \hline
\end{tabular}

${ }^{a}$ Calculated from the measured critical exponents.

bee Ref. 32.

'See Ref. 33.

${ }^{\mathrm{d}}$ This work. 


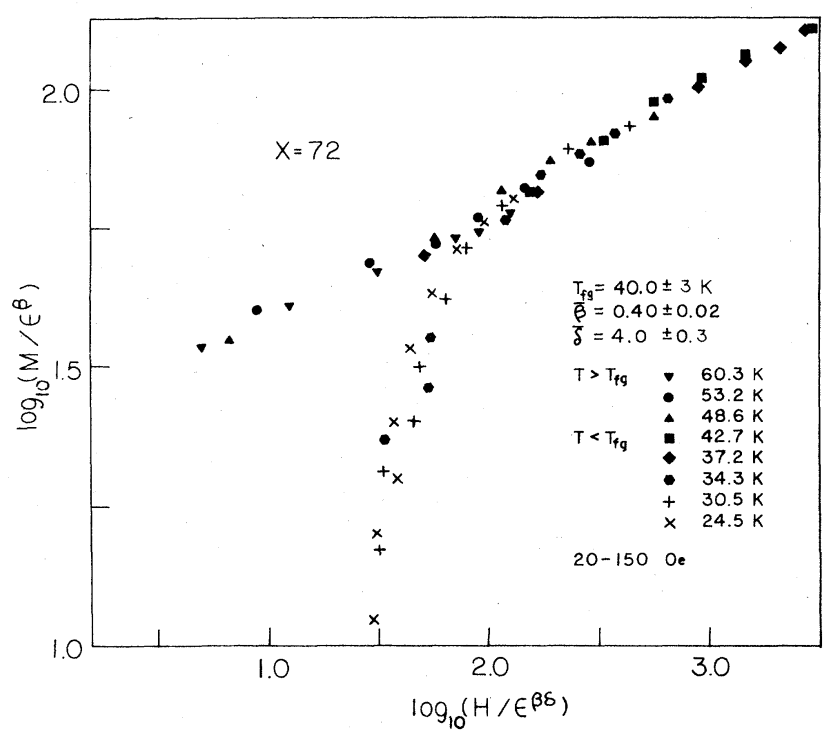

FIG. 14. Low-field magnetization versus magnetic field for the $x=72$ sample around the FM-SG transition in reduced units (see text). The data are plotted in logarithmic form to resolve the scaling at the lowest fields.

field decreases with decreasing temperature. At higher magnetic fields the isotherms cross over and assume their usual high-field behavior, with magnetization increasing with decreasing temperature, as may be seen in the insets. No particular significance should be attached to the field at which the isotherms cross over since it will depend somewhat on the temperature difference between the two isotherms chosen. The critical exponents at $T_{f g}$ are denoted by $\bar{\beta}, \bar{\delta}$, etc., in analogy to the critical exponents at $T_{p f}$. The exponents $\bar{\beta}, \bar{\delta}$ were determined in a similar way as those at $T_{p f}$. The transition temperature $T_{f g}$ was initially estimated from the peak in the out-of-phase part of the ac susceptibility and varied in the range of $\pm 4 \mathrm{~K}$ to

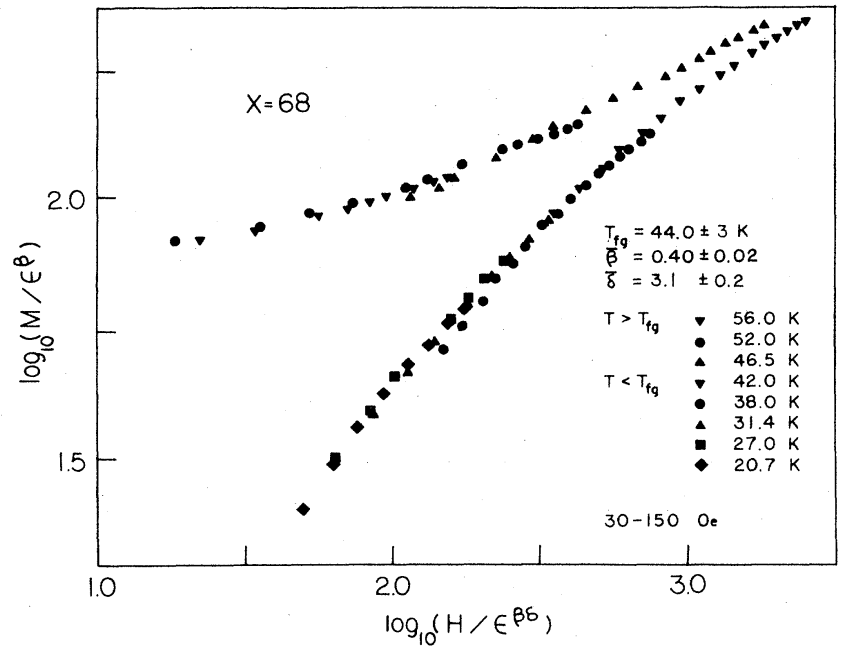

FIG. 15. Low-field magnetization versus magnetic field for the $x=68$ sample around the FM-SG transition in reduced units (see text). The data are plotted in logarithmic form to resolve the scaling at the lowest fields.

obtain best scaling. This temperature was rather well defined in terms of obtaining good scaling. The scaling behavior is shown in Figs. 14 and 15 for our $x=72$ and 68 samples at low fields. The measured exponents and transition temperatures are summarized in Table II. Although a well-defined collapse of the isotherms onto two separate curves is obtained for the $x=68$ sample, the quality of scaling is not as good as that at $T_{p f}$. The region of scaling in terms of $\epsilon$ is as large if not larger than that at $T_{p f}$. The scaling clearly does not extend to as high a field though, since the isotherms cross over at fields less than $700 \mathrm{Oe}$, and above 150 Oe large systematic deviations from scaling behavior are seen in a linear plot of $m$ versus $h$. Only an approximate collapse of the isotherms is resolved for the $x=72$ sample (Fig. 14) since the iso-

TABLE II. Experimental critical exponent values at the FM-SG transition in transition-metal and $G d$-rich metallic glasses. The symbol $G$ represents the combination $\mathrm{Ga}_{18} \mathrm{~B}_{10}$ and $G^{\prime}$ represents combinations of glass formers given in the relevant references.

\begin{tabular}{|c|c|c|c|c|}
\hline & $\bar{\beta}$ & $\bar{\gamma}$ & $\bar{\delta}$ & $T_{f g}(\mathrm{~K})$ \\
\hline$\left(\mathrm{Fe}_{1-x} \mathrm{Mn}_{x}\right)_{75} G_{25}^{\prime \text { a }}$ & & & & \\
\hline$x=0.30,0.32$ & $0.40(3)$ & & $4.5(3)$ & $31,38(2)$ \\
\hline$\left(\mathrm{Fe}_{0.2} \mathrm{Ni}_{0.8}\right)_{75} G_{25}^{\prime \mathrm{a}}$ & $0.48(6)$ & & $3.6(6)$ & $21(2)$ \\
\hline$\left(\mathrm{Co}_{0.7} \mathrm{Mn}_{0.3}\right)_{75} G_{25}^{\prime \mathrm{a}}$ & $0.38(5)$ & & $4.8(6)$ & $38(3)$ \\
\hline $\begin{array}{r}\left(\mathrm{Fe}_{x} \mathrm{Mn}_{1-x}\right)_{75} G_{25}^{\prime b} \\
x=0.65,0.70\end{array}$ & $\sim 0.40$ & $\sim 1.5$ & $\sim 4.75$ & 65,49 \\
\hline $\mathrm{Fe}_{x} \mathrm{Pd}_{82-x} \mathrm{Si}_{18}{ }^{\mathrm{c}}$ & & & & \\
\hline$x=9,10,12$ & $0.40(3)$ & & $3.5(3)$ & $18(1)^{\mathrm{e}}$ \\
\hline $\mathrm{Gd}_{72} G_{28}^{\mathrm{d}}$ & $0.40(2)$ & & $4.0(3)$ & $40(3)$ \\
\hline $\mathrm{Gd}_{68} \mathrm{La}_{4} G_{28}^{\mathrm{d}}$ & $0.40(2)$ & & $3.1(2)$ & $44(3)$ \\
\hline
\end{tabular}

asee Ref. 3.

bSee Ref. 5.

'See Ref. 6.

${ }^{\mathrm{d}}$ This work.

${ }^{\mathrm{e}}$ For the $x=10$ sample. 
therms cross over to their high-field behavior at lower fields than in the $x=68$ sample. The two branches of the scaling plot coalesce at large $h$ for the same reason. The SG phase in the $x=72$ sample is weak and these results indicate that fields $\gtrsim 100 \mathrm{Oe}$ are too large to observe the limiting critical behavior. We do not believe that the above results are due to a smeared transition. Low-field measurements below 50 Oe are required to check the scaling behavior more precisely.

\section{DISCUSSION AND CONCLUSIONS}

We have presented the first detailed results on doubletransition behavior in rare-earth-rich glassy systems. The FM order vanishes below 67 at. \% Gd, which is far from the percolation point in three dimensions, indicating the presence of significant frustration effects in the Gd-rich glasses of this work. The presence of such frustration effects may also be inferred from the slow approach to saturation at high magnetic field, and also the very presence of SG behavior, including a decrease in magnetization with decreasing temperature at low temperatures.

At the PM-FM transition we find standard scaling behavior for second-order magnetic phase transitions, and the scaling behavior is consistent with $\beta \sim 0.47$ and $\delta \sim 4.6$ along this transition line. The value of $\delta$ is close to the classical $d=3$ Heisenberg value, ${ }^{35}$ and $\beta$ is somewhat higher than the value of this model. A possible explanation for this may lie in the defect model of MullerKrumbhaar, ${ }^{36}$ and has been discussed in connection with enhanced $\beta$ values in some Gd-rich metallic glasses by Poon and Durand. ${ }^{32}$ The calculated specific-heat exponents are negative in our samples at the PM-FM transition, satisfying the criterion for sharp transition behavior.

A scaling of the magnetic isotherms was also obtained at the FM-SG transition in the $x=72$ and 68 samples. The value of $\bar{\beta}$ was $\sim 0.40$ and the value of $\bar{\delta}$ ranged from 3.1 to 4.0 - these numbers are close to those in transitionmetal glasses at the FM-SG transition. The critical exponents at the PM-FM and FM-SG transitions differ considerably, and in this sense a universality in the measured critical exponents is apparently not present. Such behavior is also found in a number of transitionmetal glasses. $^{3-6}$ de Almeida-Thouless-like transition behavior is present in the onset of hysteresis as a function of applied field in the SG phase in both the doubletransition glasses and glasses showing only PM-SG transition behavior. Such transition behavior has also been seen by Manheimer and co-workers in the reversible part of the magnetization in $\mathrm{Fe}-\mathrm{Mn}$-rich glasses. ${ }^{5}$

The presence of nonequilibrium SG behavior according to computer simulations discussed in Sec. I suggests the presence of anisotropic interactions in the Gd-rich glasses of this work. Some possibilities are a DzaloshinskyMoriya interaction ${ }^{37}$ or a weak single-ion random magnetic anisotropy as discussed in Ref. 8. We are beginning some rotation magnetization and transverse susceptibility experiments to probe the behavior of anisotropy in these systems.

\section{ACKNOWLEDGMENTS}

We thank J. Nabity and B. Richards for assistance in sample preparation and Dr. G. Muench for assistance with magnetization measurements during the latter part of this work. This work was supported by the National Science Foundation under Grant No. DMR-81-10520 at the University of Nebraska, and also by Kansas State University.
${ }^{1}$ R. Rammel and J. Souletie, in Magnetism of Metals and Alloys, edited by M. Cyrot (North-Holland, Amsterdam, 1982).

${ }^{2}$ Peter J. Ford, Contemp. Phys. 23, 141 (1982).

${ }^{3}$ Y. Yeshurun, M. B. Salamon, K. V. Rao, and H. S. Chen, Phys. Rev. B 24, 1536 (1981).

${ }^{4}$ K. V. Rao, Phys. Scr. 25, 742 (1982).

${ }^{5}$ M. A. Manheimer, S. M. Bhagat, and H. S. Chen, J. Magn. Magn. Mater. 38, 147 (1983).

${ }^{6}$ G. Dublon and Y. Yeshurun, Phys. Rev. B 25, 4899 (1982).

${ }^{7}$ M. J. O'Shea, S. G. Cornelison, Z. D. Chen, and D. J. Sellmyer, Solid State Commun. 46, 313 (1983).

${ }^{8}$ D. J. Sellmyer and M. J. O'Shea, J. Less-Common Met. 94, 59 (1983).

${ }^{9}$ M. J. O’Shea and D. J. Sellmyer, J. Appl. Phys. 53, 7722 (1982).

${ }^{10}$ S. Crane, D. W. Carnegie, and H. Claus, J. Appl. Phys. 53, 2179 (1982).

${ }^{11}$ W. Abdul-Razzaq and J. S. Kouvel, J. Appl. Phys. 55, 1623 (1984).

12A. P. Malozemoff and Y. Imry, Phys. Rev. B 24, 489 (1981).

${ }^{13}$ I. Morgenstern and K. Binder, Phys. Rev. B 22, 288 (1980).

${ }^{14}$ A. Ogielski and I. Morgenstern, J. Appl. Phys. 57, 3382 (1985); A. J. Bray and M. A. moore, J. Phys. C 17, L463 (1984); W. L. McMillan, Phys. Rev. B 29, 4026 (1984); 30,
476 (1984).

${ }^{15}$ R. W. Walstedt and L. R. Walker, Phys. Rev. Lett. 47, 1624 (1981).

${ }^{16}$ C. M. Soukoulis, K. Levin, and G. S. Grest, Phys. Rev. B 28, 1510 (1983).

${ }^{17}$ S. N. Kaul, Phys. Rev. B 24, 6550 (1981).

${ }^{18}$ P. Gaunt, S. C. Ho, Gwyn Williams, and R. W. Cochrane, Phys. Rev. B 23, 251 (1981).

${ }^{19}$ D. Sherrington and S. Kirkpatrick, Phys. Rev. Lett. 35, 1792 (1975).

20J. R. de Almeida and D. J. Thouless, J. Phys. A 11, 983 (1978).

${ }^{21}$ G. Parisi, J. Phys. A 13, L115 (1980).

${ }^{22}$ H. Sompolinsky, Phys. Rev. Lett. 47, 935 (1981).

${ }^{23}$ Y. Yeshurun, L. J. P. Ketelsen, and M. B. Salamon, Phys. Rev. B 26, 1491 (1982).

${ }^{24}$ N. Bontemps, J. Rajchenback, and R. Orbach, J. Phys. (Paris) Lett. 44, L47 (1983).

${ }^{25}$ M. Gabay and G. Toulouse, Phys. Rev. Lett. 47, 201 (1981).

${ }^{26}$ M. J. O'Shea and D. J. Sellmyer, J. Appl. Phys. 57, 3470 (1985).

${ }^{27}$ See, for example, K. Binder, Z. Phys. B 48, 319 (1982).

28 J. A. Gerber, W. L. Burnester, and D. J. Sellmyer, Rev. Sci. Instrum. 53, 691 (1982). 
${ }^{29}$ See, for example, Neil Heiman and N. Kazama, J. Appl. Phys. 49, 3 (1978).

30J. A. Geohegan and S. M. Bhagat, J. Magn. Magn. Mater. 25, 17 (1981).

${ }^{31}$ See, for example, H. E. Stanley, Introduction to Phase Transitions and Critical Phenomena (Oxford University Press, New York, 1971).

32S. J. Poon and J. Durand, Phys. Rev. B 16, 316 (1977).

33J. Durand, K. Raj, S. J. Poon, and J. I. Budrick, IEEE Trans. Magn. MAG-14, 722 (1978).
${ }^{34}$ A. Brooks Harris and T. C. Lubensky, Phys. Rev. Lett. 33, 1540 (1974).

${ }^{35}$ See G. S. Rushbrooke, G. A. Baker, Jr., and P. J. Wood, in Phase Transitions and Critical Phenomena, edited by C. Domb and M. S. Green (Academic, New York, 1974), Vol. 3, p. 245, and references therein; K. Binder, ibid., Vol. 5B, p. 1, and references therein; S. Milosevic and H. E. Stanley, Phys. Rev. B 5, 2536 (1972); 6, 986 (1972).

${ }^{36}$ H. Muller-Krumbhaar, J. Phys. C 9, 345 (1976).

${ }^{37}$ A. Fert and P. M. Levy, Phys. Rev. Lett. 44, 1538 (1980). 